\title{
The Association between Post-Stroke Depression and the Activities of Daily Living/Gait Balance in Patients with First-Onset Stroke Patients
}

\author{
Geun-Young Park ${ }^{1}$, Sun $\mathrm{Im}^{1}$, Soo-Jung Lee ${ }^{2}$, and Chi-Un Pae ${ }^{2,3 凶}$ \\ 'Department of Rehabilitation Medicine, College of Medicine, The Catholic University of Korea, Seoul, Republic of Korea \\ 2Department of Psychiatry, College of Medicine, The Catholic University of Korea, Seoul, Republic of Korea \\ ${ }^{3}$ Department of Psychiatry and Behavioural Sciences, Duke University Medical Center, Durham, NC, USA
}

This study evaluated the association between post-stroke depression (PSD) and clinical outcomes, including activities of daily living (ADL) and gait balance, in patients with first-onset stroke. One hundred and eighty inpatients were recruited and followed-up for a 6-month. The depressive, cognitive, and stroke symptoms were assessed using the Beck Depression Inventory (BDI), the Global Deterioration Scale (GDS), the modified Rankin Scale (MRS), and the Berg Balance Scale (BBS). All patients were assessed at baseline and at the end of the observation (6-month). Among 180 patients, 127 (70.6\%) were diagnosed with minimal-to-mild depression (MMD) while 53 (29.4\%) were diagnosed with moderate-to-severe depression (MSD). The odd ratio (OR) for poor outcome in the MSD group was approximately 3.7 relative to the MMD group. The proportion of patients with better balance classified by the BBS score at 6-month was significantly higher in the MMD group than in the MSD group (OR=1.375). Our findings demonstrate the potential relationship between PSD and rehabilitation outcomes measured by different rating scales in Korean stroke patients. Our study suggests that clinicians should carefully evaluate depressive symptoms in patients with stroke during routine clinical practice. Adequately-powered and wellcontrolled further studies are necessary to confirm and fully characterize this relationship.

Psychiatry Investig 2016;13(6):659-664

Key Words Post-stroke depression, Stroke, Severity, Clinical outcome, Rehabilitation.

\section{INTRODUCTION}

Post-stroke depression (PSD) is one of the most common mental complications suffered by stroke patients leading to an impaired quality of life (QoL), altered rehabilitation outcomes, and an increased mortality rate. ${ }^{1-5}$ At 3 months after a stroke, the independent predictors for PSD include a low level of education, low income, a greater severity of stroke, being younger than 68 years of age, the presence of a severe functional disability, crying behaviors, self-reported problems with activities of daily living (ADL), and the need for help during ADL. ${ }^{6,7}$

Additionally PSD significantly limits gait recovery after a

Received: April 27, 2015 Revised: June 10,2015

Accepted: July 4, 2015 Available online: October 14, 2015

$\triangle$ Correspondence: Chi-Un Pae, MD, PhD

Department of Psychiatry, Bucheon St. Mary's Hospital, College of Medicine, The Catholic University of Korea, 327 Sosa-ro, Wonmi-gu, Bucheon 14647, Republic of Korea

Tel: +82-32-340-7067, Fax: +82-32-340-2544, E-mail: pae@catholic.ac.kr

(a) This is an Open Access article distributed under the terms of the Creative Commons Attribution Non-Commercial License (http://creativecommons.org/licenses/bync/3.0) which permits unrestricted non-commercial use, distribution, and reproduction in any medium, provided the original work is properly cited. stroke, ${ }^{8,9}$ although the latency to the onset of rehabilitation treatment following a stroke does not limit motor recovery once the rehabilitation program is complete.

Few studies have investigated the association between PSD and clinical outcomes in the first-onset stroke patients, especially in the Korean population. ${ }^{10,11}$ Therefore, this study investigated the association between baseline severity of PSD and the clinical outcomes in stroke patients at 6-month after rehabilitation treatment in a naturalistic setting.

\section{METHODS}

\section{Design and subjects}

This was a 6-month observational study that included collection of socio-demographic data at baseline with personto-person interview based on case report form and the use of self- and clinician-rated scales. The post-acute phase 4-week rehabilitation program was conducted during the hospitalization period. It consisted of a complex program of universal activities that were performed five times per week.

Patients were within 2-4 weeks of hospital admission for a 
first-onset ischemic stroke. All patients were consecutively enrolled from Department of rehabilitation at Bucheon St. Mary's Hospital, The Catholic University of Korea College of Medicine, Korea between 2009 and 2011. A diagnosis of PSD was made by board-certified psychiatrists using criteria from the Diagnostic and Statistical Manual of Mental Disorders, Fourth Edition. Patients with additional Axis I psychiatric disorders other than depression were excluded to avoid any potential biases from co-morbid psychiatric disorders. The presence of co-morbid conditions was determined following an assessment of patient medication and/or clinical history by the treating physicians.

The study complied with the Declaration of Helsinki and other ethical principles regarding human experimentation. The study protocol was approved by the Institutional Review Board of Bucheon St. Mary's Hospital (IRB approval number: HC13RISI0015).

\section{Clinical outcomes}

\section{Rating scales}

All assessments were performed at baseline and at the end of the 6-month observational period. The Korean version of the Beck Depression Inventory (BDI) was used to assess the severity of depression at baseline. ${ }^{12-14}$ To evaluate the association between baseline severity of PSD and clinical outcomes a priori defined, all subjects were classified as having either minimal-to-mild depression (MMD; BDI score of $0-16$ ) or moderate-to-severe depression (MSD; BDI score $\geq 17$ ) at baseline. ${ }^{13}$ The modified Rankin Scale (MRS) $)^{15}$ was used for the evaluation of ADL. The Berg Balance Scale (BBS) was utilized to measure balance during the performance of functional tasks. ${ }^{16}$ The Global Deterioration Scale (GDS) ${ }^{17}$ was used to obtain an overview of the cognitive function.

The primary clinical outcome (good outcome) was defined using a score of 1 or 2 on the MRS score at 6-month (otherwise poor outcome). ${ }^{15}$ Additional clinical outcome used the BBS score at 6-month as following: scores of 0 to 20 for balance impairment, 21 to 40 for acceptable balance, and 41 to 56 for good balance. ${ }^{16}$ Other endpoints were the mean changes of the GDS and BBS scores from baseline to 6-month.

\section{Statistical analyses}

For all descriptive statistics, continuous variables were presented as means with standard deviations (SD), and categorical variables were presented as frequencies. To examine group differences in socio-demographic characteristics at baseline, an analysis of variance (ANOVA) or an analysis of covariance (ANCOVA) was used for continuous variables where appropriate, and a chi-square or Fisher's exact test was used for categorical variables. The correlation coefficients for baseline BDI score, change of MRS/BBS scores, and the MRS/BBS

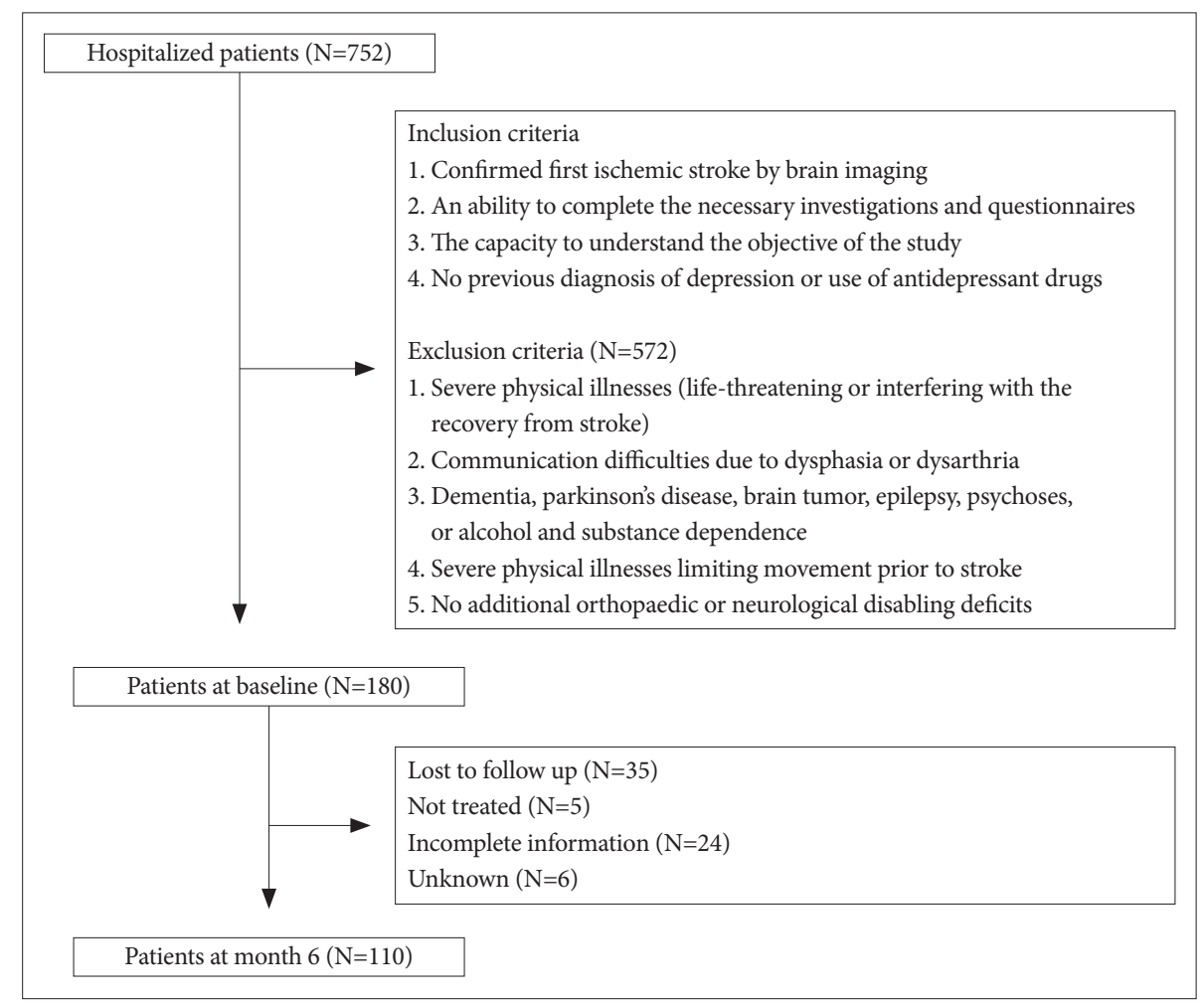

Figure 1. The patients disposition in the present study. 
scores at 6-month were also calculated.

The scores of the MMD and MSD groups were compared using ANOVA for symptoms and other clinical parameters that were reported as continuous variables. The mean changes in the BBS and GDS scores from baseline to 6-month were compared between the MMD and MSD groups using ANCOVA and age, number of co-morbid conditions, and baseline scores as covariates.

Logistic regression analysis was performed to determine the association between baseline severity of PSD (MSD) and the risk of a poor outcome as priori defined by MRS score.
Age, sex, education level, work status, family history of stroke, past history of depression, income level, alcohol use, tobacco use, marital status, number of co-morbidities, and baseline scores of BDI, BBS, GDS, and MRS were included as covariates in the regression model. In the regression model, the dependent variable was poor outcome and the independent variable was the severity of PSD (MMD vs MSD). The sensitivity, specificity, positive predictive value (PPV), negative predictive value (NPV), and number needed to diagnose (NND) of the MSD group for poor outcome were also calculated. The odds ratio (OR) and 95\% confidential intervals (CIs) were

Table 1. Clinical variables by the baseline depression severity between MMD and MSD groups

\begin{tabular}{|c|c|c|c|c|}
\hline Remark & $\operatorname{MMD}(\mathrm{N}=127)$ & $\operatorname{MSD}(\mathrm{N}=53)$ & Total $(\mathrm{N}=180)$ & $\mathrm{F}$ or $\chi^{2} / \mathrm{p}$ \\
\hline Age & $60.4(14.0)$ & $62.6(11.2)$ & $61.0(13.3)$ & NS \\
\hline \multicolumn{5}{|l|}{ Sex } \\
\hline Female & $59(46.5)$ & $33(62.3)$ & $92(51.1)$ & NS \\
\hline \multicolumn{5}{|l|}{ Education } \\
\hline$\geq$ Middle school & $89(70.0)$ & $36(67.9)$ & $115(63.9)$ & NS \\
\hline \multicolumn{5}{|l|}{ Work status } \\
\hline Employed & $85(66.9)$ & $35(66.0)$ & $110(61.1)$ & NS \\
\hline \multicolumn{5}{|l|}{ Family history of stroke } \\
\hline Yes & $19(15.0)$ & $13(24.5)$ & $32(17.8)$ & NS \\
\hline Past history of depression & $11(8.7)$ & $9(17.0)$ & $23(12.8)$ & NS \\
\hline \multicolumn{5}{|l|}{ Socioeconomic status } \\
\hline$\geq$ Middle income & $82(64.6)$ & $38(71.7)$ & $120(66.7)$ & NS \\
\hline \multicolumn{5}{|l|}{ Use of alcohol } \\
\hline Yes & $55(43.3)$ & $23(27.7)$ & $78(43.3)$ & NS \\
\hline \multicolumn{5}{|l|}{ Use of Tobacco } \\
\hline Yes & $63(49.6)$ & $27(50.9)$ & $90(50.0)$ & NS \\
\hline \multicolumn{5}{|l|}{ Marital status } \\
\hline Married & $105(82.7)$ & $47(88.7)$ & $152(84.4)$ & NS \\
\hline \multicolumn{5}{|l|}{ Comorbidity proportion } \\
\hline$\geq 1$ & $41(32.3)$ & $26(49.1)$ & $67(37.2)$ & $4.502 / 0.042$ \\
\hline Comorbidity number & $0.9(0.9)$ & $1.1(0.8)$ & $1.0(0.9)$ & NS \\
\hline \multicolumn{5}{|l|}{ Location of stroke } \\
\hline Basal ganglia & $35(27.6)$ & $14(26.4)$ & $49(27.2)$ & \\
\hline Thalamus & $21(16.5)$ & $11(20.8)$ & $32(17.8)$ & \\
\hline Pons & $14(11.0)$ & $5(9.4)$ & $19(10.6)$ & \\
\hline Cerebellum & $9(7.1)$ & $4(7.5)$ & $13(7.2)$ & \\
\hline Others & $48(37.8)$ & $19(35.8)$ & $67(37.2)$ & $0.531 / \mathrm{NS}$ \\
\hline BDI* & $7.5(4.7)$ & $27.8(9.9)$ & $12.9(11.1)$ & $300.235 /<0.0001$ \\
\hline GDS* & $3.4(1.7)$ & $4.1(1.9)$ & $3.5(1.8)$ & $4.829 / 0.03$ \\
\hline MRS* & $3.6(1.2)$ & $4.3(1.1)$ & $3.8(1.2)$ & $8.936 / 0.003$ \\
\hline BBS* & $22.9(20.9)$ & $16.3(19.3)$ & $21.0(20.6)$ & NS \\
\hline
\end{tabular}

Statistical comparison with ANOVA or Fisher Exact test. Data represent number (SD) or number (percent). *baseline scores. MMD: minimal to mild depression, MSD: moderate to severe depression, GDS: Global Deterioration Scale, MRS: modified Rankin Scale, BBS: Berg Balance Scale, MS: middle school, MI: middle income, Fhx: family history, Phx: past history, NS: not significant, BDI: Beck Depression Inventory 
calculated.

It was determined that using these statistical parameters and taking into account adjustment for covariates, the study would have a power of $80.0 \%$ to detect a medium effect of 0.4824 . This corresponded to 0.4 point difference of the change in the MRS score from baseline to 6-month between the MMD and MSD groups. All statistical analyses were conducted using the NCSS $2007^{\circledR}$ and PASS $2008^{\circledR}$ software packages (NCSS LLC, Kaysville, UT, USA). Statistical significance was two-tailed and set at $\mathrm{p}<0.05$.

\section{RESULTS}

Among 752 hospitalized patients 2009 to 2011, 572 patients did not meet the inclusion criteria and eventually 180 patients were enrolled. Among 180 inpatients, 110 patients were followed up for 6 months in which 91 and 56 patients had postbaseline MRS and BBS scores at 6-month (Figure 1). Baseline demographics classified by MMD and MSD are presented in Table 1. Among patients included, 127 (70.6\%) were diagnosed with MMD and 53 (29.4\%) were diagnosed with MSD. The mean scores of the BDI, GDS, and MRS in the MMD group were significantly lower than in the MSD group; the BBS score was higher in the MMD group than in the MSD group (Table 1).

The OR for poor outcome in the MSD group was approximately 3.7 relative to the MMD group (Table 2). The proportion of patients with better balance was significantly higher in the MMD group than in the MSD group (Table 2). The mean change of the BBS score (19.8 \pm 2.7 vs. $11.4 \pm 3.0, \mathrm{p}=0.021)$ from baseline to 6-month was significantly higher in the MMD group than in the MSD group, while significant difference in change of the GDS score ( $1.0 \pm 0.2$ vs. $1.7 \pm 0.4)$ from baseline to 6-month was not found between the two groups. Logistic regression analyses also outlined the association between baseline severity of PSD (MSD) and poor outcome ( $\mathrm{B}=1.305$, S.E. $=0.599$, Wald $=4.739, \mathrm{p}=0.029)$. The sensitivity of MSD to predict poor outcome was 0.852 , the specificity was 0.391 , the positive predictive value was 0.371 , the negative predictive value was 0.862 . The NND for poor outcome in the MSD group was 4.291 .

\section{DISCUSSION}

In the present study, the severity of PSD was significantly associated with clinical outcomes in stroke patients. The OR for poor outcome of the MSD group was approximately 3.7 relative to the MMD group. When the NPV was used as a predictor for poor outcome of the MSD group, patients with MMD had a probability of $86.2 \%$ for good outcome. Given the NND for poor outcome was 4.291 in the MSD group in stroke patients, thus using the severity of PSD (MSD) as a diagnostic screening test for poor outcome in stroke patients, it means that one out of 4.3 (10 of 43) of MSD stroke patient under-test should be diagnosed as poor outcome to rehabilitation treatment.

A consensus has not been established concerning the use of specific rating scales for the assessment of ADL as a primary endpoint since large variations in the choice of a primary endpoint have been proposed yet and the definitions of clinical outcome in stroke patients are still in debate. According to Sulter et al., ${ }^{18}$ clinical outcome should be defined utilizing a mixture of different rating scales and other related clinical factors; for instance, death, institutionalization due to stroke, a MRS score $>3$ and a Modified Barthel Index score $<60$ could be used as a combined factors for such evaluation. In addition, the cutoff scores of MRS and MBI as a main rating scale should be also established in adequately-powered and well-designed clinical trials since different clinical trials have used substantially different cut-off scores of them.

Table 2. Proportion of clinical outcomes as priori defined at 6-month between MMD and MSD groups

\begin{tabular}{lccc}
\hline & MMD & MSD & Total \\
\hline Primary clinical outcome*† & $\mathrm{N}=64$ & $\mathrm{~N}=27$ & $\mathrm{~N}=91$ \\
Good & $25(39.1)$ & $4(14.8)$ & $29(50.9)$ \\
Poor & $39(60.5)$ & $23(85.2)$ & $62(49.1)$ \\
Additional clinical outcome ${ }^{\ddagger}$ & $\mathrm{N}=38$ & $\mathrm{~N}=18$ & $\mathrm{~N}=56$ \\
Balance impairment & $5(13.2)$ & $8(44.4)$ & $13(23.2)$ \\
Acceptable balance & $11(28.9)$ & $1(5.6)$ & $12(21.4)$ \\
Good balance & $22(57.9)$ & $9(50.0)$ & $31(55.4)$
\end{tabular}

Data represent number (percent). ${ }^{*}$ good outcome=a score of 1 or 2 on the modified Rankin Scale score at 6 -month, ${ }^{\dagger}$ chi-square test, $\chi^{2}=5.142, \mathrm{p}=0.023$, odds ratio (OR) for poor outcome in $\mathrm{MSD}=3.686,95 \%$ confidence intervals (CIs), 1.033 to 14.339 , $\neq 0-20$ for balance impairment, 21-40 for acceptable balance, and 41-56 for good balance in Berg Balance Scale score at 6-month, \$Fisher's Exact test, $\chi^{2}=7.961$, $\mathrm{p}=0.022$, OR for good balance in $\mathrm{MMD}=1.375,95 \% \mathrm{CIs}, 0.386$ to 4.919. MMD: minimal to mild depression, MSD: moderate to severe depression 
Only few studies investigated the relationship between PSD and balance in stroke patients to date. One previous study found that poor balance is a principal predictor of recurrent falls by stroke patients with sensitivity and specificity values $\geq 80 \%,{ }^{19}$ while another study determined that poor balance is significantly associated with poor QoL. ${ }^{20,21}$ In one such study, subjects with a fear of falling ( $\mathrm{FoF}$ ) exhibited significantly greater levels of depressive symptoms and a lower QoL than those without FoF at baseline. ${ }^{22}$ Depressive symptoms were also an independent factor associated with falls, disabilities, previous falls, and older age in a large prospective population-based stroke incidence study with 1,104 patients. ${ }^{23}$ Similarly, our study demonstrated the potential association between baseline severity of PSD and gait balance. The baseline BBS score of the MSD group was approximately 30\% lower than that of the MMD group and the change in BBS scores from baseline to 6-month was approximately 1.7 -fold higher in the MMD group than the MSD group; both of these findings favor patients with MMD over patients with MSD. An overall shift of at least 8 points is required to reveal a genuine change in function between measures at two different points in BBS score. Thus, our findings clearly demonstrate that the severity of depression exerts a potential influence on balance in stroke patients; depression may cause lack of energy/weakness and reduce the motivation on rehabilitation leading to poor restoration of gait balance.

Our study has several strengths. We utilized the MRS that is easy and validated ADL measure tool for busy clinical practice. ${ }^{18}$ Despite balance is significantly related to falling and a poor $\mathrm{QoL}^{19}$ in stroke patients, the measurement of depression-related gait disturbances is often ignored; we used the BBS score to evaluate the association between PSD and gait disturbance. Finally, patients with comorbid psychiatric disorders other than depression were excluded from the study.

Our study has also clear limitations. A larger sample size would have provided greater sensitivity for the detection of smaller differences between the MMD and MSD groups. Moreover, the treatment duration may be insufficient to detect long-term effects of baseline severity of PSD. ${ }^{24}$ For example, the South London Stroke Register study, ${ }^{25}$ which followed patients for up to 10 years, demonstrated that depression is independently associated with poor health outcomes. Other psychological factors (i.e., personality and anxiety) may influence the clinical outcomes of stroke. A neurotic personality is associated with a lower QoL in the overall domain of stroke patients ${ }^{26}$ and anxiety has a stronger impact on QoL as a negative predictor than does depression. ${ }^{27}$ Our study employed a naturalistic observational approach, which may overlook the details of a patient's clinical course. The number of assessment was insufficient. A significant measurement loss at 6 months follow-up (i.e., BBS) should be also considered in interpretation of our findings. It should be clearly taken into mind that a cohort study cannot determine any causal relationship between certain clinical factors (i.e., depression) and disease outcomes (i.e., stroke outcome) since study design is likely to be affected by confounders as well as hidden unknown confounders. There were also significant attrition rate due to lost-to-follow up since the majority of them moved to nursing care facilities by their families, which may also bring hidden bias for study results. Finally, we have only subdivided our samples into two populations minimal to mild and moderate to severe degree using cutoff score of $16^{13}$ due to small sample size. When we had more sufficient sample size, we would have observed more elaborated relationship between depression and stoke outcomes among four-subpopulation of depression such as minimal, mild, moderate and severe depression. ${ }^{28}$ This should be taken into mind in interpretation of the results. Therefore, we have found only that potential association between PSD and stroke outcomes may exist in our patients.

Our study demonstrated the potential association between PSD and clinical outcomes of first-onset stroke in Korean patients. Early detection and management of PSD in patients with first-onset stoke should be considered during routine clinical practice. This will facilitate the tailoring of specific therapies for individual patients and aid in the determination of continued management options for the treatment of these patients.

\section{Acknowledgments}

This study was supported by a grant of the Korean Health Technology R\&D Project, Ministry of Health \& Welfare, Republic of Korea (HI12C0003).

\section{REFERENCES}

1. Williams LS, Ghose SS, Swindle RW. Depression and other mental health diagnoses increase mortality risk after ischemic stroke. Am J Psychiatry 2004;161:1090-1095.

2. Haghgoo HA, Pazuki ES, Hosseini AS, Rassafiani M. Depression, activities of daily living and quality of life in patients with stroke. J Neurol Sci 2013;328:87-91.

3. Mutai H, Furukawa T, Araki K, Misawa K, Hanihara T. Long-term outcome in stroke survivors after discharge from a convalescent rehabilitation ward. Psychiatry Clin Neurosci 2013;67:434-440.

4. Farner L, Wagle J, Engedal K, Flekkoy KM, Wyller TB, Fure B. Depressive symptoms in stroke patients: a 13 month follow-up study of patients referred to a rehabilitation unit. J Affect Disord 2010;127:211-218.

5. Dafer RM, Rao M, Shareef A, Sharma A. Poststroke depression. Top Stroke Rehabil 2008;15:13-21.

6. Sienkiewicz-Jarosz H, Milewska D, Bochynska A, Chelmniak A, Dworek N, Kasprzyk K, et al. Predictors of depressive symptoms in patients with stroke - a three-month follow-up. Neurol Neurochir Pol 2010;44:13-20.

7. Carota A, Berney A, Aybek S, Iaria G, Staub F, Ghika-Schmid F, et al. A prospective study of predictors of poststroke depression. Neurology 2005;64:428-433.

8. Huang HC, Chung KC, Lai DC, Sung SF. The impact of timing and 
dose of rehabilitation delivery on functional recovery of stroke patients. J Chin Med Assoc 2009;72:257-264.

9. Kijowski S. Difficulties in post-stroke gait improvement caused by post-stroke depression. Chin Med J (Engl) 2014;127:2085-2090.

10. Park GY, Im S, Oh CH, Lee SJ, Pae CU. The association between the severity of poststroke depression and clinical outcomes after first-onset stroke in Korean patients. Gen Hosp Psychiatry 2015;37:245-250.

11. Pae CU. Evidence-based treatment for depressive disorder. Psychiatry Investig 2015;12:278-279.

12. Beck AT, Ward CH, Mendelson M, Mock J, Erbaugh J. An inventory for measuring depression. Arch Gen Psychiatry 1961;4:561-571.

13. Jo SA, Park MH, Jo I, Ryu SH, Han C. Usefulness of Beck Depression Inventory (BDI) in the Korean elderly population. Int J Geriatr Psychiatry 2007;22:218-223.

14. Shin MS, Kim ZS, Park KB. The cut-off score for the Korean version of beck depression inventory. Korean J Clin Psychol 1993;12:71-81.

15. van Swieten JC, Koudstaal PJ, Visser MC, Schouten HJ, van Gijn J. Interobserver agreement for the assessment of handicap in stroke patients. Stroke 1988;19:604-607.

16. Berg KO, Wood-Dauphinee SL, Williams JI, Maki B. Measuring balance in the elderly: validation of an instrument. Can J Public Health 1992;83 (Suppl 2):S7-S11.

17. Reisberg B, Ferris SH, de Leon MJ, Crook T. The Global Deterioration Scale for assessment of primary degenerative dementia. Am J Psychiatry 1982;139:1136-1139.

18. Sulter G, Steen C, De Keyser J. Use of the Barthel index and modified Rankin scale in acute stroke trials. Stroke 1999;30:1538-1541.

19. Mackintosh SF, Hill KD, Dodd KJ, Goldie PA, Culham EG. Balance score and a history of falls in hospital predict recurrent falls in the 6 months following stroke rehabilitation. Arch Phys Med Rehabil 2006; 87:1583-1589.
20. Murtezani A, Hundozi H, Gashi S, Osmani T, Krasniqi V, Rama B. Factors associated with reintegration to normal living after stroke. Med Arh 2009;63:216-219.

21. Baseman S, Fisher K, Ward L, Bhattacharya A. The relationship of physical function to social integration after stroke. J Neurosci Nurs 2010;42:237-244.

22. Schmid AA, Van Puymbroeck M, Knies K, Spangler-Morris C, Watts $\mathrm{K}, \mathrm{Damush} \mathrm{T}$, et al. Fear of falling among people who have sustained a stroke: a 6-month longitudinal pilot study. Am J Occup Ther 2011;65: 125-132.

23. Kerse N, Parag V, Feigin VL, McNaughton H, Hackett ML, Bennett DA, et al. Falls after stroke: results from the Auckland Regional Community Stroke (ARCOS) Study, 2002 to 2003. Stroke 2008;39:1890-1893.

24. Jonsson AC, Delavaran H, Iwarsson S, Stahl A, Norrving B, Lindgren A. Functional status and patient-reported outcome 10 years after stroke: the Lund Stroke Register. Stroke 2014;45:1784-1790.

25. Ayerbe L, Ayis S, Crichton S, Wolfe CD, Rudd AG. The long-term outcomes of depression up to 10 years after stroke; the South London Stroke Register. J Neurol Neurosurg Psychiatry 2014;85:514-521.

26. Kim SY, Kim JM, Stewart R, Kang HJ, Kim SW, Shin IS, et al. Influences of personality traits on quality of life after stroke. Eur Neurol 2013; 69:185-192.

27. Morris JH, van Wijck F, Joice S, Donaghy M. Predicting health related quality of life 6 months after stroke: the role of anxiety and upper limb dysfunction. Disabil Rehabil 2013;35:291-299.

28. Chapman J, Oser M, Hockemeyer J, Weitlauf J, Jones S, Cheung R. Changes in depressive symptoms and impact on treatment course among hepatitis $\mathrm{C}$ patients undergoing interferon-alpha and ribavirin therapy: a prospective evaluation. Am J Gastroenterol 2011;106:21232132 . 